Cite this: Phys. Chem. Chem. Phys., 2011, 13, 8681-8689

\title{
Monitoring the effect of a control pulse on a conical intersection by time-resolved photoelectron spectroscopy
}

\author{
Yasuki Arasaki, ${ }^{a}$ Kwanghsi Wang, ${ }^{b}$ Vincent McKoy $^{b}$ and Kazuo Takatsuka ${ }^{* a}$ \\ Received 28th October 2010, Accepted 12th January 2011 \\ DOI: $10.1039 / \mathrm{c0cp02302g}$
}

We have previously shown how femtosecond angle- and energy-resolved photoelectron spectroscopy can be used to monitor quantum wavepacket bifurcation at an avoided crossing or conical intersection and also how a symmetry-allowed conical intersection can be effectively morphed into an avoided crossing by photo-induced symmetry breaking. The latter result suggests that varying the parameters of a laser to modify a conical intersection might control the rate of passage of wavepackets through such regions, providing a gating process for different chemical products. In this paper, we show with full quantum mechanical calculations that such optical control of conical intersections can actually be monitored in real time with femtosecond angle- and energy-resolved photoelectron spectroscopy. In turn, this suggests that one can optimally control the gating process at a conical intersection by monitoring the photoelectron velocity map images, which should provide far more efficient and rapid optimal control than measuring the ratio of products. To demonstrate the sensitivity of time-resolved photoelectron spectra for detecting the consequences of such optical control, as well as for monitoring how the wavepacket bifurcation is affected by the control, we report results for quantum wavepackets going through the region of the symmetry-allowed conical intersection between the first two ${ }^{2} \mathrm{~A}^{\prime}$ states of $\mathrm{NO}_{2}$ that is transformed to an avoided crossing. Geometry- and energy-dependent photoionization matrix elements are explicitly incorporated in these studies. Time-resolved photoelectron angular distributions and photoelectron images are seen to systematically reflect the effects of the control pulse.

\section{Introduction}

Nonadiabatic electronic transitions are of fundamental importance in chemistry. In particular, because a conical intersection $(\mathrm{CI})$ between two electronic states provides a very fast and efficient pathway for radiationless relaxation, ${ }^{1}$ there has been much interest in controlling transitions through a CI. Indeed, several methods have already been proposed to control the dynamical processes associated with a CI. One of these concerns the modification of electronic states involved in the CI by environmental effects of polar solvents on the PES (potential energy hypersurface) through orientational fluctuations. ${ }^{2-4}$ Another strategy is to apply a static electric field to shift the energy of a state of ionic character as in the Stark effect ${ }^{5,6}$ (see ref. 7 for the non-resonant dynamical Stark effect). More dynamical methods, which aim to suppress the transition either by preparing wavepackets that do not reach the $\mathrm{CI}^{8}$ or that destructively interfere there, ${ }^{9}$ have also been

${ }^{a}$ Department of Basic Science, Graduate School of Arts and Sciences, The University of Tokyo, Komaba, 153-8902, Tokyo, Japan.

E-mail:kaztak@mns2.c.u-tokyo.ac.jp

${ }^{b}$ A. A. Noyes Laboratory of Chemical Physics, California Institute of

Technology, Pasadena, California 91125, USA proposed. Furthermore, de Vivie-Riedle et al. have developed an optimal control theory for the dynamics associated with a CI. ${ }^{10-13}$ Recently, Lim et al. ${ }^{14}$ have used conformational changes of an excited state PES with chemical substitution to modify the pathway of the relevant nuclear (vibrational) wavepacket with respect to the location of the CI manifold.

We have recently shown in ref. 15 with full quantum wavepacket dynamics on coupled ab initio potential energy surfaces how a conical intersection can be transformed into an avoided crossing at the geometry of the CI by externally breaking its symmetry. There we showed how the population transfer through the $\mathrm{CI}$ in $\mathrm{NO}_{2}$ can be significantly suppressed using a phase-controlled far-infrared pulse. Although symmetry breaking can be caused not only by optical control but also chemically by nearby substituent groups and/or by solvent effects, ${ }^{16}$ a significant advantage of optical control is that the laser used has a number of parameters such as wavelength, intensity, pulse shape, polarization, phase, and so on, that can be externally controlled. Moreover, optical control can be applied in conjunction with the above methods based on chemical modification. The mechanism we propose here may hence have potential for external control of gating of the branching pathways for nuclear wavepackets. This study 
suggests that by varying the laser parameters, one can control the rate of passage of wavepackets through a CI.

Femtosecond time-resolved photoelectron spectroscopy is a versatile probe of ultrafast dynamics in molecules ${ }^{17-20}$ and is particularly well suited for studies of wavepacket dynamics in nonadiabatic systems where nuclear and electronic modes are coupled. In fact, internal conversion in polyatomic molecules was among the earliest suggested application of this technique, ${ }^{21}$ which was subsequently realized experimentally. ${ }^{22}$ We have also previously demonstrated the utility of time-resolved photoelectron spectra, along with the need to incorporate geometry- and energy-dependent photoionization matrix elements in such studies, in a series of papers tracking wavepacket dynamics in different scenarios: vibrational motion across a one-dimensional double-well potential in an excited state of $\mathrm{Na}_{2},{ }^{23-27}$ wavepacket bifurcation at an avoided crossing in $\mathrm{NaI},{ }^{28,29}$ and proton transfer in the ground state of chloromalonaldehyde. ${ }^{30-32}$ Anticipating that advances in ultrashort pulse shaping technology may also well enable the observation of wavepacket dynamics through a CI on the actual time scale of the nonadiabatic transition, we recently studied the photoelectron energy and angular distributions expected in and around the CI between the lowest ${ }^{2} \mathrm{~A}^{\prime}$ states of the $\mathrm{NO}_{2}$ molecule ${ }^{33,34}$ and showed that the photoelectron signals, particularly the angular distributions, provide a valuable window on wavepacket dynamics there. Exploiting the results of these two studies, ${ }^{15,33,34}$ here we show with full quantum mechanical calculations that such optical control of a conical intersection can actually be monitored in real time with femtosecond angle- and energy-resolved photoelectron spectroscopy. This suggests that one can optimally control the above gating process through the conical intersection by a feedback loop based on varying the control parameters and monitoring the photoelectron velocity map images. This strategy should provide a far more efficient and rapid means of optimal control than measuring the ratio of products.

To explore the sensitivity of the photoelectron spectrum to the proposed scheme for optical control of a CI and how wavepacket bifurcation might be affected by such control, we report results for quantum wavepackets passing through the symmetry-allowed conical intersection between the first two ${ }^{2} \mathrm{~A}^{\prime}$ states of $\mathrm{NO}_{2}$, which is transformed into an avoided crossing with a far infrared pulse. The CI between the first two ${ }^{2} \mathrm{~A}^{\prime}$ states of the $\mathrm{NO}_{2}$ molecule is known to lead to an extremely complex absorption spectra, ${ }^{35-37}$ and has been the subject of numerous studies of nonadiabatic dynamics. ${ }^{38-44}$ For $C_{2 v}$ geometries the two surfaces $\left({ }^{2} \mathrm{~A}_{1}\right.$ and $\left.{ }^{2} \mathrm{~B}_{2}\right)$ intersect at a bond angle that depends on the bond length and form a one-dimensional CI seam. ${ }^{39}$ The seam is located close to the bottom of the excited state and is readily accessible by a vibrational wavepacket launched onto the excited electronic surface from the Franck-Condon region of the ground state.

The scheme for our studies in $\mathrm{NO}_{2}$ is illustrated in Fig. 1. $\mathrm{NO}_{2}$ molecules in the ground vibrational level are assumed to be transiently aligned beforehand, possibly by using short laser pulses. ${ }^{45,46}$ The molecule is then pumped to an excited electronic state by a femtosecond pulse. Because of the ultrafast time-scale of the associated dynamics, we employ pulses with a full-width at half-maximum (FWHM) of $8 \mathrm{fs}$ in these studies.

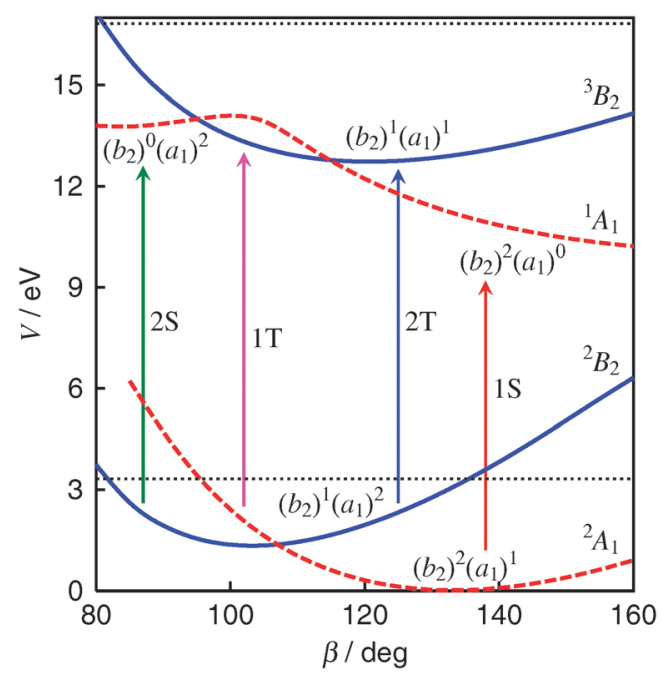

Fig. 1 A one-dimensional section along the bond angle $\beta$ (bond lengths $r_{1}=r_{2}=1.22 \AA$ ) for the potential surfaces relevant to the control and observation of the ${ }^{2} A_{1} /{ }^{2} B_{2} \mathrm{CI}$ in $\mathrm{NO}_{2} \cdot{ }^{15,34}$ The two neutral surfaces are shown in the diabatic representation and the lowest singlet and triplet ion surfaces in the adiabatic representation. The four possible ionization channels between these surfaces (1T for state 1 to triplet, $2 \mathrm{~S}$ for state 2 to singlet ionization, and so on) are indicated with arrows and the dominant electron configurations are shown along each potential curve. Dotted horizontal lines indicate the energy reached by the pump $\left(v_{0}+\omega_{\mathrm{pu}}=3.3\right)$ and probe $\left(v_{0}+\omega_{\mathrm{pu}}+\omega_{\mathrm{pr}}=\right.$ $16.8 \mathrm{eV})$.

Wavepacket motion on the excited state, as well as on the ground electronic state that is coupled to the excited state by the $\mathrm{CI}$, is probed with a time-delayed femtosecond pulse that directly ionizes the molecule. The photoelectrons are then energy- and angle-resolved for signatures of the wavepacket motion. A control pulse with an FWHM of 16 fs, but of very low frequency so that its field amplitude varies on the time scale for passage of the wavepacket through the CI, is applied between the pump and probe pulses to modify the excited state dynamics. To simulate the photoelectron spectra we numerically time-evolve the wavepackets on the relevant electronic surfaces in all three dimensions (neglecting rotation), employing the diabatic representation to handle the nonadiabatic interaction at the CI. The coupling of the electronic surfaces due to the pump and probe pulses is explicitly included in the Hamiltonian, and geometry- and energy-dependent photoionization matrix elements are employed throughout.

The remainder of this paper is organized as follows. Section 2 outlines the theoretical formulation used in this work and the computational methods and details are discussed in Section 3. The time-dependent photoelectron spectra of the wavepackets in and around the CI and the effects of the control pulse on these spectra are discussed in Section 4. Section 5 concludes the paper.

\section{Formulation}

\subsection{Transformation of a symmetry-allowed conical intersection to an avoided crossing}

The description of our control scheme for transformation of a symmetry-allowed conical intersection to an avoided crossing 
in the region of the $\mathrm{CI}$ begins with a two-state model. The Hamiltonian for $n$ nuclei in a molecular system with a nonadiabatic interaction between two electronic states can be written in the diabatic representation as

$$
\hat{H}=\hat{T}_{\boldsymbol{R}} \mathbf{1}+\mathbf{V}(\boldsymbol{R}),
$$

where $\hat{T}_{\boldsymbol{R}}$ is the kinetic energy operator for the nuclear coordinates $\boldsymbol{R}, \mathbf{1}$ the unit matrix, and the diabatic potential energy matrix

$$
\mathbf{V}(\boldsymbol{R})=\left(\begin{array}{cc}
V_{1}(\boldsymbol{R}) & V_{12}(\boldsymbol{R}) \\
V_{12}(\boldsymbol{R}) & V_{2}(\boldsymbol{R})
\end{array}\right),
$$

contains the potential energy surfaces $V_{1}(\boldsymbol{R})$ and $V_{2}(\boldsymbol{R})$ for the two diabatic electronic states 1 and 2, and the diabatic coupling element $V_{12}(\boldsymbol{R})$ between them. For a CI, $V_{1}(\boldsymbol{R})=$ $V_{2}(\boldsymbol{R})$ and $V_{12}(\boldsymbol{R})=0$, thereby forming a $(3 n-8)$-dimensional intersection space. Applying a perturbation $\mathbf{V}_{E}(\boldsymbol{R}, t)$, which can also be represented as a two dimensional matrix and may be time dependent, one can modify the property and geometry of the CI manifold. $\mathbf{V}_{E}(\boldsymbol{R}, t)$ might be due to environmental effects of solvents or a functional group adjacent to the location of the CI which would change the force field at the CI. The perturbation may also be more conveniently introduced externally in the form of a linearly polarized pulsed optical field, which is the case treated here.

Consider the dynamics of a molecule in which two coupled electronic states belong to the same irreducible representation in an initial molecular geometry (an asymmetric triangular geometry for instance), thus resulting in level repulsion. As the molecule changes its geometry and acquires a higher symmetry (say, an isosceles triangle), the two electronic states can come to belong to different irreducible representations, resulting in degeneracy. It is around such a geometry that we can prevent the raising of the symmetry by applying an external optical field and inducing an additional coupling between the two electronic states of different irreducible representations (i.e. breaking local symmetry). The two states will then continue to undergo level repulsion as in an ordinary avoided crossing. To be more concrete, $\mathbf{V}_{E}(\boldsymbol{R}, t)$ might be

$$
\mathbf{V}_{E}(\boldsymbol{R}, t)=-\left(\begin{array}{ll}
\mu_{11}(\boldsymbol{R}) & \mu_{12}(\boldsymbol{R}) \\
\mu_{12}(\boldsymbol{R}) & \mu_{22}(\boldsymbol{R})
\end{array}\right) E(t),
$$

where $E(t)$ is the electric field in a particular direction, $\mu_{11}(\boldsymbol{R})$ and $\mu_{22}(\boldsymbol{R})$ the diabatic dipole moments along this direction, and $\mu_{12}(\boldsymbol{R})$ the transition dipole moment in the same direction. At the geometry of the CI, $\boldsymbol{R}_{X}$, the diabatic coupling potential $V_{12}\left(\boldsymbol{R}_{X}\right)$ is by definition zero. On the other hand, one may be able to choose a direction of the applied laser field so that $\mu_{12}\left(\boldsymbol{R}_{X}\right)$ is nonzero, and this is generally possible by a simple symmetry consideration. Diagonalization of the Hamiltonian of eqn (1) with the potential term replaced by $\mathbf{V}(\boldsymbol{R})+\mathbf{V}_{E}(\boldsymbol{R}, t)$ results in the instantaneous "adiabatic" potential energy matrix, $\tilde{\mathbf{V}}(\boldsymbol{R}, t)$, where degeneracy at the geometry of the CI is lifted. Specifically, the potential energy surfaces at the CI geometry will become separated by a gap of $\Delta \tilde{V}\left(\boldsymbol{R}_{X}\right)=$ $2\left|\mu_{12}\left(\boldsymbol{R}_{X}\right) E(t)\right|$. The overall effect of the external field is, in fact, to shift the CI away from its original location.
Nevertheless, in the very short time of passage for a single wavepacket through the CI region, the trajectory cannot adjust to the changing shape of the potential energy surfaces and the wavepacket continues to go through the original CI region. Thus, an avoided crossing is, in effect, formed at the original geometry of the conical intersection and nonadiabatic population transfer is expected to be greatly suppressed there.

\subsection{Time-resolved photoelectron spectroscopy}

Our formulation of time-resolved pump-probe photoelectron spectroscopy has been fully discussed in earlier papers ${ }^{24,28}$ and here we present just a brief outline with emphasis on the use of geometry- and energy-dependent photoionization matrix elements.

The wavefunction of the total system, $\Psi(\boldsymbol{r}, \boldsymbol{R}, t)$, is expanded in the electronic wavefunctions relevant to the pump-probe arrangement,

$$
\begin{aligned}
\psi(\boldsymbol{r}, \boldsymbol{R}, t)= & \sum_{i=X, A} \chi_{i}(\boldsymbol{R}, t) \Phi_{i}(\boldsymbol{r} ; \boldsymbol{R}) \\
& +\int \mathrm{d} \boldsymbol{k} \chi_{\boldsymbol{k}}(\boldsymbol{R}, t) \Phi_{\boldsymbol{k}}^{(-)}(\boldsymbol{r} ; \boldsymbol{R}),
\end{aligned}
$$

where $i=X, A$ labels the adiabatic neutral electronic wavefunctions and $\Phi_{k}^{(-)}(\boldsymbol{r} ; \boldsymbol{R})$ is the wavefunction of the final state (ion plus photoelectron). The latter is labeled with the continuous photoelectron wave vector $\boldsymbol{k}$, and $\boldsymbol{R}$ is the set of internal nuclear coordinates, $\boldsymbol{r}$ the electronic coordinates, and $t$ time. Because of the ultrafast time scale of the vibrational dynamics of interest here, and also because of the large bandwidth of the ultrashort pulses employed, molecular rotation is neglected in this study. Thus, $\chi_{i}(\boldsymbol{R}, t)$ and $\chi_{\boldsymbol{k}}(\boldsymbol{R}, t)$ are identified with vibrational wavefunctions in the neutral and ionized systems, respectively.

Coupled equations for the vibrational wavefunctions can then be written as

$$
\begin{aligned}
i \frac{\partial}{\partial t}\left(\begin{array}{c}
\chi_{X} \\
\chi_{A}
\end{array}\right)= & {\left[\hat{\mathbf{T}}+\left(\begin{array}{cc}
V_{X}(\boldsymbol{R}) & V_{\mathrm{pu}}(\boldsymbol{R}, t) \\
V_{\mathrm{pu}}(\boldsymbol{R}, t) & V_{A}(\boldsymbol{R})
\end{array}\right)\right]\left(\begin{array}{c}
\chi_{X} \\
\chi_{A}
\end{array}\right) } \\
& +\int \mathrm{d} \mathbf{k}\left(\begin{array}{c}
V_{\mathrm{pr}}^{(X) *}(\boldsymbol{R}, t) \\
V_{\mathrm{pr}}^{(A) *}(\boldsymbol{R}, t)
\end{array}\right) \chi_{\boldsymbol{k}},
\end{aligned}
$$

and

$$
i \frac{\partial}{\partial t} \chi_{\boldsymbol{k}}=\left[\hat{T}_{\boldsymbol{R}}+V_{\mathrm{ion}}(\boldsymbol{R})+\varepsilon_{k}\right] \chi_{\boldsymbol{k}}+\sum_{i=X, A} V_{\mathrm{pr}}^{(i)} \chi_{i},
$$

where atomic units are used throughout. $V_{\text {ion }}(\boldsymbol{R})$ is the potential energy surface of the molecular ion and $\varepsilon_{k}$ is the photoelectron energy (labeled by the photoelectron wave number $k$ ). $\hat{\mathbf{T}}$ is the $2 \times 2$ kinetic energy matrix operator in the adiabatic electronic basis, where off-diagonal terms appear because of the nonadiabatic coupling $(\mathrm{CI})$ between the two electronic states. For ease of computation, the system is converted to an appropriate quasidiabatic representation where the coupling of the surfaces enters through the potential energy term and the kinetic energy operator is made diagonal. In this work, the phenomenological method of Hirsch et al. ${ }^{39}$ 
is used to obtain the quasidiabatic surfaces. $V_{\text {pu }}(\boldsymbol{R}, t)$ is the pump pulse interaction coupling the ground and excited neutral states in the dipole approximation

$$
V_{\mathrm{pu}}(\boldsymbol{R}, t)=-E_{\mathrm{pu}} f_{\mathrm{pu}}(t) \cos \left(\omega_{\mathrm{pu}} t\right) \mu_{12}(\boldsymbol{R}),
$$

where $E_{\mathrm{pu}}$ is the strength of the pump field, $f_{\mathrm{pu}}$ the pump pulse envelope, $\omega_{\mathrm{pu}}$ the pump frequency, and $\mu_{12}(\boldsymbol{R})$ the transition dipole amplitude along the polarization of the pump pulse. The complex function $V_{\mathrm{pr}}^{(i)}(\boldsymbol{k}, \boldsymbol{R}, t, \Delta T, \hat{\Omega})$ is the probe pulse interaction which will be discussed further shortly. This interaction is specified by the neutral electronic surface being probed, $i$, the delay time from the center of pump pulse, $\Delta T$, and the angles between the probe pulse polarization and the molecular axis, $\hat{\Omega}$.

The electronic wavefunction of the final state is written as an antisymmetrized product of a cation wavefunction, $\Phi_{+}(\boldsymbol{r} ; \boldsymbol{R})$, and a photoelectron orbital, $\phi_{\boldsymbol{k}}^{(-)}(\boldsymbol{r} ; \boldsymbol{R})$,

$$
\Phi_{\boldsymbol{k}}^{(-)}(\boldsymbol{r} ; \boldsymbol{R})=\mathscr{A}\left(\Phi_{+}(\boldsymbol{r} ; \boldsymbol{R}) \cdot \phi_{\boldsymbol{k}}^{(-)}(\boldsymbol{r} ; \boldsymbol{R})\right),
$$

and $\phi_{\boldsymbol{k}}^{(-)}(\boldsymbol{r} ; \boldsymbol{R})$ is expanded in spherical harmonics, $Y_{l \lambda}(\hat{k})$, with $\hat{k}$ being the angular part of $\boldsymbol{k}$,

$$
\phi_{\boldsymbol{k}}^{(-)}(\boldsymbol{r} ; \boldsymbol{R})=\sum_{l \lambda} i^{l} \mathrm{e}^{-i \eta_{l}} Y_{l \lambda}^{*}(\hat{k}) \psi_{k l \lambda}^{(-)}(\boldsymbol{r} ; \boldsymbol{R}) .
$$

In eqn (9), $\boldsymbol{r}$ indicates the electronic coordinates in the molecular frame, $\psi_{k l \lambda}^{(-)}(\boldsymbol{r} ; \boldsymbol{R})$ is a partial wave component of the photoelectron orbital in the molecular frame with momentum $k, \lambda$ the projection of $l$ in the molecular frame, and $\eta_{l}$ the Coulomb phase shift. ${ }^{47}$

In the dipole approximation, the probe interaction, $V_{\mathrm{pr}}$, becomes

$$
V_{\mathrm{pr}}=-E_{\mathrm{pr}} f_{\mathrm{pr}}(t-\Delta T) \cos \left(\omega_{\mathrm{pr}} t\right) D,
$$

where the dipole operator, $D$, is

$$
D=\sqrt{\frac{4 \pi}{3}} r \sum_{\mu} D_{\mu 0}^{1}(\hat{\Omega}) Y_{1 \mu}(\hat{r}),
$$

for the linearly polarized case. Here $E_{\mathrm{pr}}$ is the probe field strength, $f_{\mathrm{pr}}(t-\Delta T)$ the probe pulse envelope, $\omega_{\mathrm{pr}}$ the probe frequency, $\Delta T$ the delay time from the center of the pump pulse, $r$ and $\hat{r}$ the magnitude and angular part of $\boldsymbol{r}$, respectively, and the angles $\hat{\Omega}$ orient the probe polarization with respect to the molecule through the rotation matrix $\left(\mathscr{D}_{\mu 0}^{1}\right)$.

The probe pulse interaction, $V_{\mathrm{pr}}^{(i)}(\boldsymbol{k}, \boldsymbol{R}, t, \Delta T, \hat{\Omega})$, for ionization of the state $i$ then has the form

$$
\begin{aligned}
V_{\mathrm{pr}}^{(i)}(\boldsymbol{k}, \boldsymbol{R}, t, \Delta T, \hat{\Omega})= & \left\langle\Phi_{\boldsymbol{k}}^{(-)}(\boldsymbol{r} ; \boldsymbol{R})\left|V_{\mathrm{pr}}\right| \Phi_{i}(\boldsymbol{r} ; \boldsymbol{R})\right\rangle_{\boldsymbol{r}} \\
= & -E_{\mathrm{pr}} f_{\mathrm{pr}}(t-\Delta T) \cos \left(\omega_{\mathrm{pr}} t\right) \\
& \times \sum_{l \lambda} C_{l \lambda}^{(i)}(k, \boldsymbol{R}, \hat{\Omega}) Y_{l \lambda}(\hat{k}),
\end{aligned}
$$

where

$$
C_{l \lambda}^{(i)}(k, \boldsymbol{R}, \hat{\Omega})=\sqrt{\frac{4 \pi}{3}} \sum_{\mu} I_{l \lambda \mu}^{(i)}(k, \boldsymbol{R}) D_{\mu 0}^{1}(\hat{\Omega}),
$$

and the bracket subscript $\boldsymbol{r}$ denotes integration over only the electronic coordinates. $I_{I \lambda \mu}^{(i)}(k, \boldsymbol{R})$ are partial wave matrix elements in the molecular frame formed from dipole matrix elements between $\mid \Phi_{+} \psi(k l \lambda)$ and the components of the wavefunction $\Phi_{i}$. These $C_{l \lambda}^{(i)}(\boldsymbol{k}, \boldsymbol{R}, \hat{\Omega})$ coefficients provide a geometry- and energy-dependent description of the photoionization process.

The ion vibrational wavefunction, $\chi_{\boldsymbol{k}}(\mathbf{R}, t)$, is also expanded in spherical harmonics as

$$
\chi_{\boldsymbol{k}}(\boldsymbol{R}, t)=\sum_{l \lambda} \chi_{k l \lambda}(\boldsymbol{R}, t) Y_{l \lambda}(\hat{k}),
$$

and integration over $\boldsymbol{k}$ in eqn (5) becomes an integration over $k$ and summation over $l$ and $\lambda$. Integration over $k$ is handled by a quadrature (with weights $w_{j}$ ) over discrete points $k_{j}$ $\left(j=1,2, \ldots, N_{k}\right)$ and the integration is terminated at some maximum wave number $k_{N_{k}}$. With discretization of both the wave number and angle, the ion vibrational wavefunction is represented by a set of wavefunctions $\left\{\chi_{k_{i} l \lambda}(\boldsymbol{R}, t)\right\}$, each associated with different photoelectron energies and angles. For $N_{l}$ sets of $(l, \lambda)$ included in the calculation, the number of coupled equations of motion is thus $\left(2+N_{k} N_{l}\right)$ for the two neutral states and the discretized final state. Eqn (5) and (6) are then discretized and solved numerically.

After propagation of the vibrational wavepackets for a delay time $\Delta T$, the final ion population, $P_{\text {ion }}(\Delta T)$, can be obtained by integrating over $\boldsymbol{k}$,

$$
\begin{aligned}
P_{\text {ion }}= & \int \mathrm{d} \boldsymbol{k} \int \mathrm{d} \boldsymbol{R}\left|\chi_{\boldsymbol{k}}\left(\boldsymbol{R}, t_{\mathrm{f}}\right)\right|^{2} \\
\approx & \sum_{j=1}^{N_{k}} w_{j} k_{j}^{2} \int_{0}^{2 \pi} \mathrm{d} \phi_{k} \int_{0}^{\pi} \sin \theta_{k} \mathrm{~d} \theta_{k} \int \mathrm{d} \boldsymbol{R} \\
& \times\left|\sum_{l \lambda} \chi_{k_{j} l \lambda}\left(\boldsymbol{R}, t_{\mathrm{f}}\right) Y_{l \lambda}\left(\theta_{k}, \phi_{k}\right)\right|^{2} \\
\equiv & \sum_{j=1}^{N_{k}} w_{j} \int_{0}^{2 \pi} \mathrm{d} \phi_{k} \int_{0}^{\pi} \sin \theta_{k} \mathrm{~d} \theta_{k} P_{k_{j}}\left(\theta_{k}, \phi_{k}\right) \\
\equiv & \sum_{j=1}^{N_{k}} w_{j} k_{j} P\left(\varepsilon_{k_{j}}\right),
\end{aligned}
$$

where $t_{\mathrm{f}}$ is the time after the probe pulse interaction is over and $\hat{k}=\left(\theta_{k}, \phi_{k}\right)$. The photoelectron kinetic energy distribution, $P\left(\varepsilon_{k}\right)$, and energy-resolved molecular frame photoelectron angular distribution, $P_{k_{j}}\left(\theta_{k}, \phi_{k}\right)$ are given by the integrands of eqn (15).

The angular coordinates $\left(\theta_{k}, \phi_{k}\right)$ and the ion partial wave functions can be transformed to a frame aligned with the probe polarization,

$$
\tilde{\chi}_{l m}=\sum_{\lambda} D_{m \lambda}^{l}(\hat{\Omega}) \chi_{l \lambda},
$$

where the tilde over variables denotes the transformed frame. Integration over the $\tilde{\phi}$ angle around the polarization axis of 
the probe results in averaged angular distributions of the form:

$$
\begin{aligned}
P_{k_{j}}\left(\tilde{\theta}_{k}\right) & =k_{j}^{2} \int_{0}^{2 \pi} \mathrm{d} \tilde{\phi}_{k} \int \mathrm{d} \boldsymbol{R}\left|\sum_{l m} \tilde{\chi}_{k_{j} l m} Y_{l m}\left(\tilde{\theta}_{k}, \tilde{\phi}_{k}\right)\right|^{2} \\
& =k_{j}^{2} \int \mathrm{d} \boldsymbol{R}\left|\sum_{l m} \tilde{\chi}_{k_{j} l m} Y_{l m}\left(\tilde{\theta}_{k}, 0\right)\right|^{2} .
\end{aligned}
$$

This averaging results in angular distributions for a set of molecules with their molecular axes aligned along one direction.

\section{Computation}

The $a b$ initio potential surfaces used and the method of wavepacket propagation are described in previous papers. ${ }^{15,34}$ Briefly, the surfaces were obtained from a state-averaged complete active space self-consistent field calculation as implemented in the MOLPRO quantum chemistry package, ${ }^{4-50}$ using Dunning's correlation consistent polarized triple zeta (cc-pVTZ) basis set, ${ }^{51}$ and interpolated over a dense grid of points. The ground and excited neutral states were diabatized with the phenomenological method of Hirsch et $a l .,{ }^{39}$ in which the diagonal elements of the dipole moment matrix in the direction of the bond angle bisector are minimized. Note that for the symmetric molecule where the diabatic surfaces collectively coincide with the adiabatic, these dipole moment components are zero. The singlet and triplet ion states were obtained with the same method and basis set as the neutral states but were not state-averaged. They were, however, shifted vertically up by 1.9 and $1.7 \mathrm{eV}$, respectively, to bring them into agreement with experiment. Photoionization matrix elements were obtained assuming a frozen-core Hartree-Fock model for the final state wavefunctions (ion plus photoelectron) in which the ion orbitals are taken to be those of the neutral core, and the photoelectron orbital is obtained as a solution of a one-electron Schrödinger equation containing the Hartree-Fock potential of the molecular ion ${ }^{47}$

$$
\left(-\frac{1}{2} \nabla^{2}+V_{\text {ion }}(\boldsymbol{r} ; \boldsymbol{R})-\varepsilon_{k}\right) \psi_{k l \lambda}^{(-)}(\boldsymbol{r} ; \boldsymbol{R})=0 .
$$

The vibrational wavefunctions were represented on a fast Fourier transform grid in Jacobi coordinates, $(r, R, \theta)$, where $r$ denotes the distance between $\mathrm{N}$ and one of the $\mathrm{O}$ atoms, and the distance $R$ and the angle $\theta$ define the vector from the center-of-mass of the NO moiety to the other $\mathrm{O}$ atom, and numerically propagated with the split operator short-time propagator method. ${ }^{52-55}$

Fig. 1 shows a one-dimensional section of potential surfaces relevant to the present scheme. The two neutral surfaces are shown in the diabatic representation and the lowest singlet and triplet ion surfaces in the adiabatic representation. The four possible ionization channels between these surfaces are indicated with arrows. The dominant electron configurations of the wavefunction are shown along each potential curve and dotted horizontal lines indicate the energy reached by the pump and probe pulses. In $C_{2 \mathrm{v}}$ geometry the dominant configurations of the diabatic neutral states 1 and 2 are $\cdots\left(4 b_{2}\right)^{2}\left(6 a_{1}\right)^{1}$ and $\cdots\left(4 b_{2}\right)^{1}\left(6 a_{1}\right)^{2}$, respectively. Ionization from either the $4 b_{2}$ orbital of state 1 or the $6 a_{1}$ orbital of state 2 leads to the triplet ion. We hereafter refer to these channels as 1T (ionization of state 1 to the triplet ion) and $2 \mathrm{~T}$ (ionization of state 2 to the triplet ion). The singlet ion state has a dominant configuration of $\cdots\left(4 b_{2}\right)^{2}\left(6 a_{1}\right)^{0}$ for bond angles larger than $100^{\circ}$, which changes to $\cdots\left(4 b_{2}\right)^{0}\left(6 a_{1}\right)^{2}$ for smaller bond angles. Therefore for bond angles larger than $100^{\circ}$, only the neutral state 1 can ionize to the singlet ion, and we refer to this channel as $1 \mathrm{~S}$. For bond angles less than $100^{\circ}$, only the neutral state 2 can ionize to the singlet ion, and we refer to this channel as $2 \mathrm{~S}$. Note that for ionization to the triplet ion, the $1 \mathrm{~T}$ and $2 \mathrm{~T}$ channels are open for all bond angles, in contrast to the case of ionization to the singlet ion.

The molecule is taken to lie in the $x y$-plane, and for $C_{2 \mathrm{v}}$ geometry, the two $\mathrm{O}$ atoms lie parallel to the $x$-axis and the $\mathrm{N}$ atom lies on the negative $y$ axis. The pump pulse is polarized along the $x$-axis and the polarization of the probe is taken parallel to that of the pump. We further assume that the molecules are initially transiently aligned using short laser pulses. $^{45,46}$ The propagation of the vibrational wavefunction is done entirely in internal (Jacobi) coordinates, and the molecular orientation enters into the calculation only through the matrix elements of the pump and probe couplings. The internal coordinates are converted to bond angle $(\beta)$ and bond lengths $\left(r_{1}\right.$ and $\left.r_{2}\right)$ for presentation. The system is propagated from the lowest vibrational level of the ground electronic state with a time step $\Delta t$ of $0.1 \mathrm{fs}$. The excited state wavepacket is generated with a pump pulse of $\omega_{\mathrm{pu}}=3.1 \mathrm{eV}$ and a Gaussian envelope with an FWHM of $8 \mathrm{fs}$. The center of the pump pulse is taken as time $t=0 \mathrm{fs}$.

To transform the conical intersection into an avoided crossing, we use a control pulse of the form ${ }^{56}$

$$
\begin{aligned}
E(t)= & E_{0} f\left(t-t_{0}\right) \cos \left(\omega\left(t-t_{0}\right)+\phi\right) \\
& +\frac{E_{0}}{\omega} \frac{\mathrm{d} f}{\mathrm{~d} t} \sin \left(\omega\left(t-t_{0}\right)+\phi\right),
\end{aligned}
$$

where the field intensity is $3.2 \times 10^{13} \mathrm{~W} \mathrm{~cm}^{-2}$, the frequency is $\omega=0.1 \mathrm{eV}$, the center of pulse is at $t_{0}=8 \mathrm{fs}$, the phase is $\phi=0$, and the FWHM is $16 \mathrm{fs}$. The second term is included in eqn (19) because the time derivative of the pulse envelope is not small compared to the frequency of the control pulse. The parameters are chosen so that the shape of the field approximately matches that of the half-cycle pulse used in the previous paper ${ }^{15}$ between 4 and $12 \mathrm{fs}$ when the excited state wavepacket is located in the conical intersection region. This choice results in the same dynamics as in ref. 15. The pulse width is short compared to its oscillation period and the phase is such that the maximum of the wave matches that of the pulse envelope, and the shape of the resulting field is very similar to the pulse envelope (other choices were briefly described in the supplement to the previous paper ${ }^{15}$ ). Fig. 2 illustrates the control pulse and its effect on the potential energy surfaces. The solid curve in Fig. 2(c) shows the shape of the control pulse field. The field is polarized along the direction perpendicular to the bond angle bisector for $C_{2 \mathrm{v}}$ geometry and belongs to the irreducible representation $B_{2}$, and upon application the two potential surfaces of $A_{1}$ and $B_{2}$ character belong to the same irreducible representation $\left(\mathrm{A}^{\prime}\right)$. At its peak 

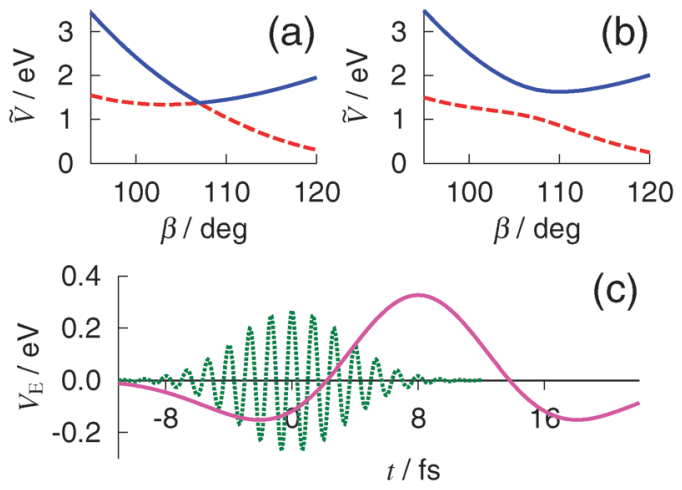

Fig. 2 (a) The conical intersection without the control pulse and (b) level repulsion of the potential surfaces at the maximum of the control pulse ( $8 \mathrm{fs})$. (c) Shape of the control pulse (solid curve). The off-diagonal element of $\mathbf{V}_{E}(\boldsymbol{R}, t)$ for the geometry of the conical intersection in units of $\mathrm{eV}$. The shape of the pump pulse (dotted curve) at the global minimum geometry is also shown for comparison.

( $t=8 \mathrm{fs}$ ) the control pulse splits the levels at the CI by $\sim 0.6 \mathrm{eV}$, as shown in Fig. 2(b). Polarizability terms that may be quantitatively significant ${ }^{57}$ are neglected in this study. The shape of the pump pulse is shown with dashed lines in Fig. 2(c) for comparison.

Photoelectron spectra of the wavepackets with the pump and control pulses were then obtained for various delay times, $\Delta T$, for a probe pulse with $\omega_{\mathrm{pr}}=13.5 \mathrm{eV}$ and an FWHM of 8 fs. Photoelectron spectra were obtained separately for ionization to the singlet and triplet ion states.

\section{Results and discussion}

\subsection{Wavepacket dynamics with the control pulse}

Fig. 3 shows the time evolution of the population of the diabatic state 2 . The dashed curve (a) shows the population without the control pulse and the solid curve (b) shows the population when the control pulse is applied. During the FWHM 8 fs pump pulse, the state 2 population builds up to $\sim 0.5$ and then decreases as the wavepacket passes through the $\mathrm{CI}$ region around $8 \mathrm{fs}$. The state 1 population created in the first passage through the CI returns to the CI region for the second time around $20 \mathrm{fs}$ and results in a population transfer in the reverse direction. Comparison of the curves in Fig. 3

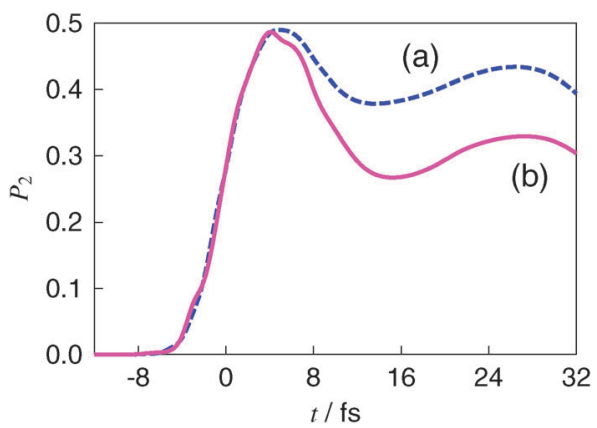

Fig. 3 Time evolution of state 2 population following the pump pulse. The population with the control pulse (solid) and that without the control pulse (dashed) are shown. shows that application of the control pulse centered at $t=8 \mathrm{fs}$ enhances population transfer from diabatic state 2 to state 1 . At later times when the control pulse becomes insignificant, the populations for the two cases evolve in the same way with time. An adiabatic picture of similar controlled population dynamics was discussed in the previous paper. ${ }^{15}$

Fig. 4 shows the evolution of the wavepackets with time increasing from left to right. The top frames (a) show the results without application of the control pulse, and the bottom frames (b) the results with the control pulse on. Each column shows a projection of the wavefunction onto the $\left(\beta, r_{1}\right)$ plane at times indicated at the top of the panels. Blue shade shows the wavepacket component on diabatic state 2 , and red shade shows the component on diabatic state 1 . Potential energy contours for $r_{2}=1.37 \AA$ are shown for 3.0 (outer contour) and $1.8 \mathrm{eV}$ (inner contours) with the same colors. A cross $(\times)$ indicates the position of the CI for the same bond length in the upper row panels; in the lower row, the intersection of the $V_{1}=V_{2}$ and $V_{12}-\mu_{12} E(t)=0$ surfaces (pink vertical and horizontal curves, respectively) is shown for the same bond length and represents the position of the conical intersection under the effect of the control pulse. The portion of the initial wavefunction left unexcited on state 1 by the pump pulse has been removed for clarity in Fig. 4. Similar figures (for the case without control) were shown in previous papers; ${ }^{33,34}$ the current figures have higher thresholds for plotting compared to previous figures to emphasize differences in heights for different spatial regions.

Without the control pulse [Fig. 4(a)], there is no transfer between diabatic states at the center of the wavepacket $\left(r_{1}=r_{2}\right)$ and the center of the wavepacket goes through the $\mathrm{CI}$, where the interaction potential between diabatic states is zero $\left(V_{12}=0\right.$ at the $\left.\mathrm{CI}\right)$. Thus, population transfer from the initially excited state 2 to state 1 occurs away from the position of the CI and symmetrically for $r_{1}<r_{2}$ and $r_{1}>r_{2}$. There are thus two regions of state 1 population at $8 \mathrm{fs}$. After passage through the region of $\mathrm{CI}$, the state 2 wavepacket component reaches its small angle turning point by $20 \mathrm{fs}$, while the state 1 wavepacket is reflected earlier by its steeper inner well compared to state 2 . By $20 \mathrm{fs}$, the state 1 component is moving quickly through the $\mathrm{CI}$ region for the second time, resulting in some transfer of population from state 1 to state 2 . The state 1 component in the plot at $20 \mathrm{fs}$ is shown much smaller compared to that at $16 \mathrm{fs}$ because the wavepacket at $20 \mathrm{fs}$ is much flatter and is spread over a large region of space compared to when the wavepacket peaks at its turning point at $16 \mathrm{fs}$.

When the control pulse is applied [Fig. 4(b)], the CI is effectively lifted and there is population transfer between diabatic states even at the center of the wavepacket. The fastest component of the wavepacket reaches the CI before the surfaces are shifted by the control pulse and behaves in a similar fashion to the case without the control pulse. However, the bulk of the population that is transferred no longer splits into two components, and because of the asymmetry introduced by the control pulse, the resulting wavepacket is also asymmetric with respect to the two bond lengths. The behavior of the conical intersection under the control pulse results in more state 1 and less state 2 population after the first passage 

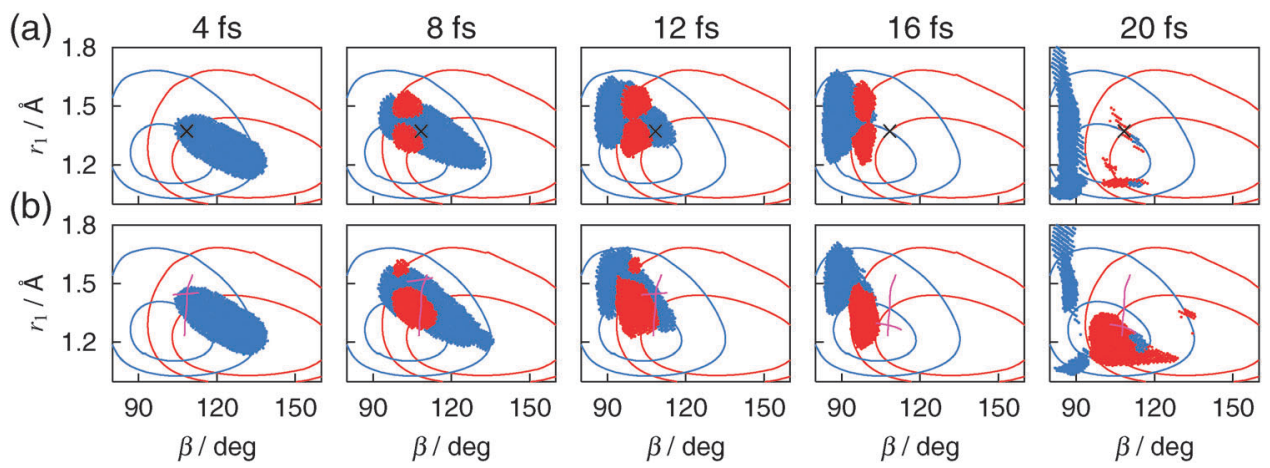

Fig. 4 Time evolution of the wavepacket formed by the pump pulse, (a) without and (b) with application of the control pulse. Projections of the diabatic wavepacket amplitude onto the $\left(\beta, r_{1}\right)$ plane are shown for selected times $t$ indicated at the top. Wavepacket components on state 1 and 2 are indicated with red and blue shades, respectively. Potential contours for 3.0 and $1.8 \mathrm{eV}$ at $r_{2}=1.37 \AA$ are also shown. In the upper row, the position of the $\mathrm{CI}$ is indicated with a cross $(\times)$, while in the lower row, the intersection of the $V_{1}=V_{2}$ and $V_{12}-\mu_{12} E(t)=0$ surfaces (pink vertical and horizontal curves, respectively) is shown for $r_{2}=1.37 \AA$, and represents the position of the perturbed conical intersection.

through the region of the CI, as is evident from comparison of the two cases at $20 \mathrm{fs}$ (rightmost panel).

\subsection{Time-resolved photoelectron angular distributions reflecting the controlled dynamics}

Energy-resolved photoelectron angular distributions [eqn (17)] were computed for probing of the wavepackets in Fig. 4: (a) without the control pulse, $P_{k_{j}}^{(\mathrm{a})}\left(\tilde{\theta}_{k}\right)$, and (b) with the control pulse, $P_{k_{j}}^{(\mathrm{b})}\left(\tilde{\theta}_{k}\right)$. Fig. 5(a) shows the time evolution of $P_{k_{j}}^{(\mathrm{a})}\left(\tilde{\theta}_{k}\right)$ as photoelectron velocity map images. The delay time between the pump and probe increases from left to right for $\Delta T=4$, 12 , and $20 \mathrm{fs}$, representing times before reaching the $\mathrm{CI}$ region, after the first passage through the CI region, and when the state 2 component reaches the small angle turning point, respectively. The axes in each frame represent photoelectron momenta in atomic units and the momentum along the polarization of the pump and probe is shown on the vertical axis (parallel to $\mathrm{O}-\mathrm{O}$ for $C_{2 \mathrm{v}}$ geometry). Brightness indicates the signal strength $P_{k_{j}}^{(\mathrm{a})}\left(\tilde{\theta}_{k}\right)$ and the numbers on the rightmost scale box are given in units of $10^{-4}$. A similar figure was given
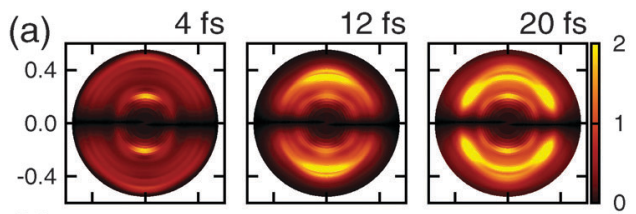

(b)
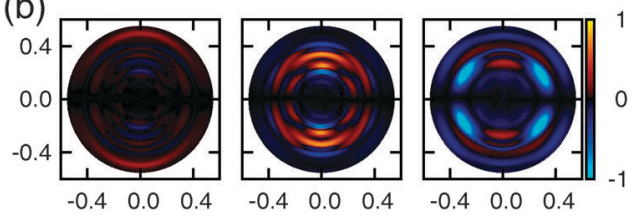

Fig. 5 (a) Femtosecond time-resolved photoelectron velocity map images without the control pulse for delay times $\Delta T=4$ (before reaching the CI region), 12 (after passage through the CI region), and 20 fs (state 2 component reaching the small angle turning point). (b) Difference between the photoelectron velocity map images with and without the control pulse. The photoelectron momentum in atomic units is indicated along the axes. The polarization axes of the pump and probe are vertical. Numbers on the rightmost scale box are given in units of $10^{-4}$. in Fig. 7 of the previous paper. ${ }^{34}$ As the wavepacket passes through the CI region for the first time (between 4 and $12 \mathrm{fs}$ ) a signal in the direction parallel to the pump and probe polarization develops. At later times when the wavepacket on diabatic state 2 reaches the turning point at small angles, the $2 \mathrm{~S}$ channel becomes active and a strong signal in the diagonal direction develops.

The difference between the energy-resolved photoelectron angular distributions with and without the control pulse, $\Delta P_{k_{j}}\left(\tilde{\theta}_{k}\right)=P_{k_{j}}^{(\mathrm{b})}\left(\tilde{\theta}_{k}\right)-P_{k_{j}}^{(\mathrm{a})}\left(\tilde{\theta}_{k}\right)$, is plotted in Fig. 5(b) as velocity map images. Red brightness indicates a more intense signal with the control pulse while blue brightness indicates a less intense signal with the control pulse. At 4 fs (leftmost), before the control pulse has significant amplitude, the difference between the two cases is negligible. After the first passage through the CI region (12 fs), the relative populations of the two diabatic states have been modified by the control pulse and an increase in signal for the region around $k=0.35$ $(1.7 \mathrm{eV})$ and a decrease for higher and lower momenta are seen in the difference images [middle frame of Fig. 5(b)]. At later times [rightmost frame of Fig. 5(b)], the signal from the $2 \mathrm{~S}$ channel is much reduced by the control pulse because there is less population in state 2 reaching the small angle region for this ionization channel.

To provide some insight into the velocity maps of Fig. 5, Fig. 6 shows the time evolution of the photoelectron angular distribution for $\varepsilon_{k}=1.7 \mathrm{eV}$. This energy corresponds to a value of $k=0.35$ a.u. in the 12 fs frame of Fig. 5 where the signal with the control pulse on is larger than the signal without the control pulse. The top row (a) in Fig. 6 shows the angular distributions obtained without the control pulse, $P_{k_{j}}^{(\mathrm{a})}\left(\tilde{\theta}_{k}\right)$, while the bottom row (b) shows the angular distributions with the control pulse on, $P_{k_{j}}^{(\mathrm{b})}\left(\tilde{\theta}_{k}\right)$. The delay time between the pump and probe increases from left to right. For each frame, the polarization of the pump and probe lies along the vertical axis and the distributions are cylindrically symmetric around this axis because of the averaging of eqn (17). The distributions are also symmetric with respect to the horizontal axis because the probe pulse does not differentiate between molecules with $r_{1}>r_{2}$ and $r_{1}<r_{2}$. The angular distribution for each frame is normalized 
(a)
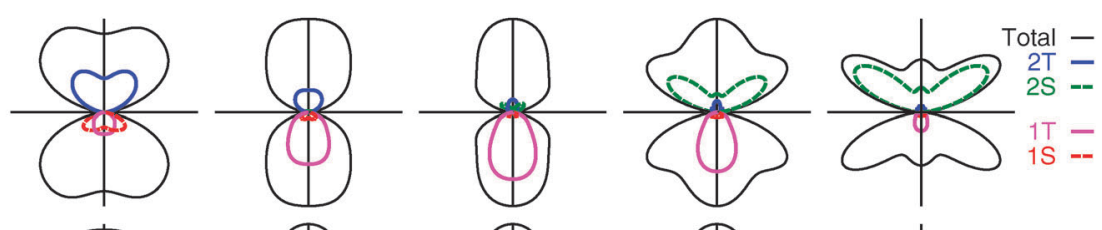

(b)
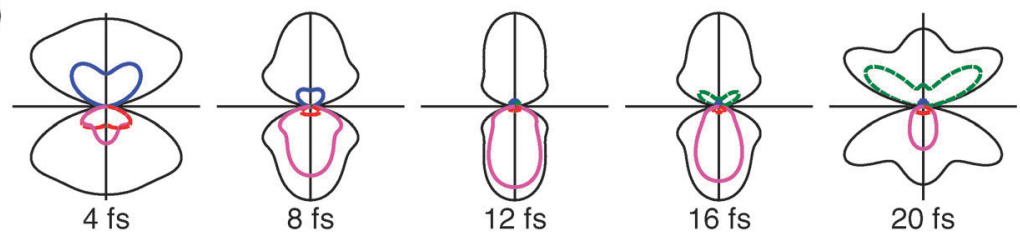

Fig. 6 Time evolution of the photoelectron angular distributions for a kinetic energy of $\varepsilon_{k}=1.7 \mathrm{eV}$ : (a) without and (b) with the control pulse. The polarization axes of the pump and probe are vertical.

separately for each delay time for clearer presentation of the overall shape; information on the relative signal strengths between different delay times is omitted. Inside the solid black contour of the photoelectron angular distribution for each frame, the contribution from each ionization channel is plotted in color. The top half of each frame shows the $2 \mathrm{~T}$ (blue, solid) and 2S (green, dashed) channel components and the bottom half shows the 1T (pink, solid) and 1S (red, dashed) channel components, although all components are actually symmetric with respect to the horizontal axis. A similar figure was given for other photoelectron energies for the same system and pulses, but without the control pulse, in Fig. 6 of the previous paper. ${ }^{34}$

Fig. 6 shows that the overall time evolution of the photoelectron images in Fig. 5 is mainly due to the change in the relative composition of the components of the signal. During the first passage through the CI region (between 4 and $12 \mathrm{fs}$ ), the $2 \mathrm{~T}$ component signal shrinks while the $1 \mathrm{~T}$ component signal grows, reflecting the transfer of population between the diabatic states. For later times (16 and $20 \mathrm{fs}$ ), a strong $2 \mathrm{~S}$ component signal develops as the wavepacket on diabatic state 2 reaches the turning point at small angles. These overall changes in the photoelectron angular distributions were noted in the previous paper ${ }^{34}$ without a control pulse.

The difference between the photoelectron angular distributions with the control pulse on [Fig. 6(b)] and without the control pulse [Fig. 6(a)] mainly reflects the change in the composition of the photoelectron signal due to the control pulse. With the control pulse on, there is somewhat more state 1 population from the first passage through the CI region centered at $8 \mathrm{fs}$, resulting in a larger 1T component and a smaller $2 \mathrm{~T}$ component for subsequent times ( $12 \mathrm{fs}$ ), and a smaller $2 \mathrm{~S}$ component at later times ( $20 \mathrm{fs})$. This is the main reason for the increase/decrease of signal strength seen in the time evolution of the difference photoelectron velocity images in Fig. 5(b).

Most of the component signals themselves do not change shape over time. The exceptions are the $2 \mathrm{~T}$ channel signal during passage through the CI region, and the 1T channel signal during the same time, but only with the control pulse on. In the previous paper, ${ }^{34}$ it was noted for the $2 \mathrm{~T}$ channel signal that, when the wavepacket has amplitude on both sides of the $\mathrm{CI}$, the signal resulting from that region changes shape during passage through the region. In contrast, the state 1 component of the wavepacket appears only after the wavepacket has gone through the CI region. Because it exists only on one side of the $\mathrm{CI}$, the angular distribution for the 1T channel did not change during the time of the first CI passage.

With the control pulse, there is much greater transfer between the diabatic states before the wavepacket reaches the CI position. Thus the wavepacket component on state 1 is formed both just before and after first passage through the $\mathrm{CI}$ region, and hence the $1 \mathrm{~T}$ channel component signal changes shape during this time. The photoelectron angular distribution from the state 1 component before reaching the CI position has protrusions out to the sides [4 and 8 fs frames of row (b) of Fig. 6] in contrast to the distributions parallel to the polarization axis for the state 1 component on the other side of the CI position ( $12 \mathrm{fs}$ and later). These protrusions out to the sides survive even in the difference map images at $12 \mathrm{fs}$ in Fig. 5(b). This is an example where the time evolution of the photoelectron angular distribution reflects not only the composition of the evolving component signals, but also the change in shape of the angular distribution of a single component signal from one side of the CI to the other. This behavior can hardly be seen in the aggregate signal without the application of the control pulse.

\section{Conclusions}

We have explored the application of femtosecond timeresolved photoelectron spectroscopy for real-time monitoring of wavepacket dynamics around the CI between the first two ${ }^{2} \mathrm{~A}^{\prime}$ states of $\mathrm{NO}_{2}$ for the case when the $\mathrm{CI}$ has been transformed to an avoided crossing by an external field. Suppression of the nonadiabatic interaction at the CI by the control pulse changes the composition of the vibrational wavepacket going through the $\mathrm{CI}$ and these changes are reflected in the evolution of time-resolved photoelectron angular distributions calculated using ab initio geometryand energy-dependent photoionization amplitudes. Changes in the composition of the wavepacket, reflected in the relative contributions of different ionization channels, and the effect of the geometry dependence of the photoionization amplitudes on photoelectron angular distributions are both seen to play a role. Time-resolved photoelectron spectroscopy is thus seen to be useful in tracking the modification of the dynamics by a control pulse. Furthermore, the results suggest that such a 
control pulse can provide additional information on the dynamics at the conical intersection.

A combination of the methodologies of such optical control at conical intersections and the photoionization-spectroscopic observation of the wavepacket in these regions can enable studies of the control and monitoring of the gating process through a conical intersection. This will naturally lead to an efficient and unique method of optimal control by the tuning of the parameters of the control laser and the monitoring of subsequent changes. Thus our study also suggests that timeresolved photoelectron spectroscopy may be utilized as a sensitive tool to explore how nature might control conical intersections in optical systems.

\section{Acknowledgements}

This work was supported in part by a Grant-in-Aid for Basic Science from the Ministry of Education, Culture, Sports, Science and Technology of Japan. VM and KW acknowledge support by the National Science Foundation under grant CHE-0956610. These studies also made use of the resources of the Jet Propulsion Laboratory's Supercomputing and Visualization Facility.

\section{References}

1 Conical Intersections: Electronic Structure, Dynamics and Spectroscopy, ed. W. Domcke, D. R. Yarkony and H. Köppel, World Scientific, Singapore, 2004.

2 I. Burghardt, L. S. Cederbaum and J. T. Hynes, Faraday Discuss., 2004, 127, 395-411.

3 I. Burghardt and J. T. Hynes, J. Phys. Chem. A, 2006, 110, 11411-11423.

4 S. Alfalah, O. Deeb, S. Zilberg and Y. Haas, Chem. Phys. Lett., 2008, 459, 100-104.

5 M. Squillacote, J. Wang and J. Chen, J. Am. Chem. Soc., 2004, 126, 1940-1941.

6 X. F. Xu, A. Kahan, S. Zilberg and Y. Haas, J. Phys. Chem. A, 2009, 113, 9779-9791.

7 B. J. Sussman, M. Y. Ivanov and A. Stolow, Phys. Rev. A: At., Mol., Opt. Phys., 2005, 71, 051401; B. J. Sussman, D. Townsend, M. Y. Ivanov and A. Stolow, Science, 2006, 314, 278-281.

8 R. Mitrić, M. Hartmann, J. Pittner and V. Bonačić-Koutecký, J. Phys. Chem. A, 2002, 106, 10477-10481.

9 M. Abe, Y. Ohtsuki, Y. Fujimura, Z. Lan and W. Domcke, J. Chem. Phys., 2006, 124, 224316.

10 R. de Vivie-Riedle, L. Kurtz and A. Hofmann, Pure Appl. Chem., 2001, 73, 525-528.

11 D. Geppert, A. Hofmann and R. de Vivie-Riedle, J. Chem. Phys., 2003, 119, 5901-5906.

12 R. de Vivie-Riedle and A. Hofmann, in Conical Intersections: Electronic Structure, Dynamics and Spectroscopy, ed. W. Domcke, D. R. Yarkony and H. Köppel, World Scientific, Singapore, 2004, ch. 18.

13 D. Geppert and R. de Vivie-Riedle, Chem. Phys. Lett., 2005, 404, 289-295.

14 J. S. Lim, Y. S. Lee and S. K. Kim, Angew. Chem., Int. Ed., 2008, 47, 1853-1856.

15 Y. Arasaki and K. Takatsuka, Phys. Chem. Chem. Phys., 2010, 12, $1239-1242$.

16 V. Deckert, K. Iwata and H. Hamaguchi, J. Photochem. Photobiol., $A, 1996, \mathbf{1 0 2}, 35-38$.

17 I. V. Hertel and W. Radloff, Rep. Prog. Phys., 2006, 69, 1897-2003.

18 T. Suzuki, Annu. Rev. Phys. Chem., 2006, 57, 555-592.

19 M. Wollenhaupt, V. Engel and T. Baumert, Annu. Rev. Phys. Chem., 2005, 56, 25-56.

20 A. Stolow, A. E. Bragg and D. M. Neumark, Chem. Rev., 2004, 104, 1719-1758.
21 M. Seel and W. Domcke, J. Chem. Phys., 1991, 95, 7806-7822.

22 V. Blanchet, M. Zgierski, T. Seidemann and A. Stolow, Nature, 1999, 401, 52-54.

23 Y. Arasaki, K. Takatsuka, K. Wang and V. McKoy, Chem. Phys. Lett., 1999, 302, 363-374.

24 Y. Arasaki, K. Takatsuka, K. Wang and V. McKoy, J. Chem. Phys., 2000, 112, 8871-8884.

25 K. Takatsuka, Y. Arasaki, K. Wang and V. McKoy, Faraday Discuss., 2000, 115, 1-15.

26 Y. Arasaki, K. Takatsuka, K. Wang and V. McKoy, J. Electron Spectrosc. Relat. Phenom., 2000, 108, 89-98.

27 Y. Arasaki, K. Takatsuka, K. Wang and V. McKoy, J. Chem. Phys., 2001, 114, 7941-7950.

28 Y. Arasaki, K. Takatsuka, K. Wang and V. McKoy, Phys. Rev. Lett., 2003, 90, 248303.

29 Y. Arasaki, K. Takatsuka, K. Wang and V. McKoy, J. Chem. Phys., 2003, 119, 7913-7923.

30 Y. Arasaki, K. Yamazaki, M. T. do N. Varella and K. Takatsuka, Chem. Phys., 2005, 311, 255-268.

31 M. T. do N. Varella, Y. Arasaki, H. Ushiyama, V. McKoy and K. Takatsuka, J. Chem. Phys., 2006, 124, 154302.

32 M. T. do N. Varella, Y. Arasaki, H. Ushiyama, K. Takatsuka, K. Wang and V. McKoy, J. Chem. Phys., 2007, 126, 054303.

33 Y. Arasaki and K. Takatsuka, Chem. Phys., 2007, 338, 175-185.

34 Y. Arasaki, K. Takatsuka, K. Wang and V. McKoy, J. Chem. Phys., 2010, 132, 124307.

35 A. Delon, R. Jost and M. Lombardi, J. Chem. Phys., 1991, 95, 5701-5718.

36 A. Delon and R. Jost, J. Chem. Phys., 1999, 110, 4300-4308.

37 A. Delon, R. Jost and M. Jacon, J. Chem. Phys., 2001, 114, 331-344.

38 E. Haller, H. Köppel and L. S. Cederbaum, J. Mol. Spectrosc., 1985, 111, 377-397.

39 G. Hirsch, R. J. Buenker and C. Petrongolo, Mol. Phys., 1990, 70, $835-848$.

40 U. Manthe and H. Köppel, J. Chem. Phys., 1990, 93, 1658-1669.

41 S. Mahapatra, H. Köppel, L. S. Cederbaum, P. Stampfuß and W. Wenzel, Chem. Phys., 2000, 259, 211-226.

42 V. Kurkal, P. Fleurat-Lessard and R. Schinke, J. Chem. Phys., 2003, 119, 1489-1501.

43 A. T. J. B. Eppink, B. J. Whitaker, E. Gloaguen, B. Soep, A. M. Coroiu and D. H. Parker, J. Chem. Phys., 2004, 121, 7776-7783.

44 M. Sanrey and M. Joyeux, J. Chem. Phys., 2006, 125, 014304.

45 C. Z. Bisgaard, O. J. Clarkin, G. Wu, A. M. D. Lee, O. Geßner, C. C. Hayden and A. Stolow, Science, 2009, 323, 1464-1468.

46 F. Rosca-Pruna and M. J. J. Vrakking, Phys. Rev. Lett., 2001, 87, 153902.

47 R. R. Lucchese, D. K. Watson and V. McKoy, Phys. Rev. A: At., Mol., Opt. Phys., 1980, 22, 421-426; R. R. Lucchese, G. Raseev and V. McKoy, Phys. Rev. A: At., Mol., Opt. Phys., 1982, 25, 2572-2587; S. N. Dixit and V. McKoy, J. Chem. Phys., 1985, 82, 3546-3553; R. R. Lucchese, K. Takatsuka and V. McKoy, Phys. Rep., 1986, 131, 147-221; K. Wang and V. McKoy, J. Chem. Phys., 1991, 95, 4977-4985; K. Wang and V. McKoy, Annu. Rev. Phys. Chem., 1995, 46, 275-304.

48 H.-J. Werner and P. J. Knowles, J. Chem. Phys., 1985, 82, $5053-5063$.

49 P. J. Knowles and H.-J. Werner, Chem. Phys. Lett., 1985, 115, 259-267.

50 R. D. Amos, A. Bernhardsson, A. Berning, P. Celani, D. L. Cooper, M. J. O. Deegan, A. J. Dobbyn, F. Eckert, C. Hampel, G. Hetzer, P. J. Knowles, T. Korona, R. Lindh, A. W. Lloyd, S. J. McNicholas, F. R. Manby, W. Meyer, M. E. Mura, A. Nicklass, P. Palmieri, R. Pitzer, G. Rauhut, M. Schütz, U. Schumann, H. Stoll, A. J. Stone, R. Tarroni, T. Thorsteinsson and H.-J. Werner, MOLPRO, a package of ab initio programs, designed by H.-J. Werner and P. J. Knowles, Version 2006.1, 2006.

51 T. H. Dunning, J. Chem. Phys., 1989, 90, 1007-1023.

52 M. D. Feit, J. A. Fleck and A. Steiger, J. Comput. Phys., 1982, 47, 412-433.

53 J. Alvarellos and H. Metiu, J. Chem. Phys., 1988, 88, 4957-4966.

54 R. Kosloff, J. Phys. Chem., 1988, 92, 2087-2100.

55 C. E. Dateo and H. Metiu, J. Chem. Phys., 1991, 95, 7392-7400.

56 N. Došlić, J. Phys. Chem. A, 2006, 110, 12400-12405.

57 A. D. Bandrauk, E.-W. S. Sedik and C. F. Matta, J. Chem. Phys., 2004, 121, 7764-7775. 